\title{
The spectroscopic orbit and the geometry of R Aquarii
}

\author{
M. Gromadzki ${ }^{1}$ and J. Mikołajewska ${ }^{1}$
}

\author{
Nicolaus Copernicus Astronomical Center, Bartycka 18, 00-716 Warszawa, Poland \\ e-mail: [marg;mikolaj]@camk.edu.pl
}

Received 25 April 2008 / Accepted 4 December 2008

\begin{abstract}
Context. R Aqr is one of the closest symbiotic binaries and the only D-type system with radial velocity data suitable for orbital parameter estimation.

Aims. The aims of our study are to derive a reliable spectroscopic orbit of the Mira component, and to establish connections between the orbital motion and other phenomena exhibited by R Aqr.

Methods. We reanalyze and revise the velocity data compiled by McIntosh \& Rustan complemented by additional velocities.

Results. We find an eccentric orbit $(e=0.25)$ with a period $43.6 \mathrm{yr}$. This solution is in agreement with a resolved VLA observation of this system. We demonstrate that the last increase in extinction towards the Mira variable in 1974-1981 occurred during its superior, spectroscopic conjunction, and can be due to obscuration by a neutral material in the accreting stream. We also show that jet ejection is not connected with the orbital position.
\end{abstract}

Key words. stars: binaries: symbiotic - stars: binaries: spectroscopic - stars: individual: R Aquarii - stars: fundamental parameters

\section{Introduction}

$\mathrm{R}$ Aqr is a symbiotic binary surrounded by an hourglass nebula (Solf \& Ulrich 1985), and both its cool and hot components are unique. The cool component is a Mira variable with a pulsation period of $387^{\mathrm{d}}$ with $\mathrm{SiO}$ and $\mathrm{H}_{2} \mathrm{O}$ maser emission, which is rare, with only three among 48 symbiotics with Miras exhibiting this emission (Whitelock 2003). The hot component shows sporadic, jet ejections (e.g. Kellogg et al. 2007; Nichols et al. 2007), and Nichols et al. (2007) also detected a 1734 s periodic oscillation in Chandra X-ray observations, which suggested that the hot companion is a magnetic white dwarf. Willson et al. (1981) interpreted a brightness reduction in pulsation maxima as the eclipsing of the Mira by a companion surrounded by an extended gas cloud. This interpretation was supported by two facts. First, during the last hypothetical eclipse (1974-1980), a minimum was observed in the near-IR light curve similar to that corresponding to dust obscuration in other symbiotic Miras (e.g., Whitelock et al. 1983). Secondly, a spectrum obtained during the first such event (1886-1894) exhibits only faint emission lines of hydrogen and no trace of $\mathrm{M}$ type spectral features (Townley et al. 1928). In the historical, visual, light curve, three phases of reduced maxima are present spaced at $44 \mathrm{yr}$ intervals and this value was adopted by Willson et al. (1981) as the orbital period. Additionally, the $\mathrm{O}-\mathrm{C}$ diagram showed variation with a period of 22 yr. Wallerstein (1986) used a number of spectral absorption lines associated with the Mira in an attempt to determine the orbital elements of R Aqr. His results were consistent with the $44 \mathrm{yr}$ period, but uncertain. Hinkle et al. (1989) combined these archival data with radial velocities (RV) determined from near-IR CO and Ti I lines, and obtained a $44 \mathrm{yr}$ period and a highly eccentric, $e=0.6$, orbital solution

McIntosh \& Rustan (2007) collected radial velocity data of $\mathrm{SiO}$ maser emission of R Aqr (Lepine et al. 1978; Cohen \& Ghigo 1980; Spencer et al. 1981; Lane 1982; Hall et al. 1990; Cho et al. 1996; Boboltz 1997; Hollis et al. 2000; Alcolea et al. 1999; Pardo et al. 2004; McIntosh \& Rustan 2007) complemented by previous radial velocities derived from optical absorption lines (Merrill 1950; Jacobsen \& Wallerstein 1975; Wallerstein 1986) and near-IR CO and Ti I lines (Hinkle et al. 1989). All of these features are associated with the cool component or material in its close neighborhood and reflect its motion around the mass center. In astrophysical masers, emission originating in many maser spots moves around the Mira variable. Spectra of masers exhibit a series of emission lines with various velocities associated with different maser spots. McIntosh (2006) studied changes in the velocity centroid (VC) of $\mathrm{SiO}$ maser emission from 76 single evolved giants, and showed that the mean difference in the VCs measured at different epochs was only $0.065 \pm 2.00 \mathrm{~km} \mathrm{~s}^{-1}$, and that the $\mathrm{VC}$ of the emission distribution can be used to derive the velocity of the star. Based on this result, McIntosh \& Rustan (2007) adopted the VC in their work for all kinds of velocity data analysis.

McIntosh \& Rustan (2007) also estimated orbital elements using a program developed by Gudehus (2001). They inferred an eccentric orbit, $e=0.52$, with a period $34.6 \mathrm{yr}$. Their orbital period was significantly shorter than any previous estimates as well as the period derived in our present study. Their other were of reasonable accuracy if we interpret their system mass, equal to $0.043 M_{\odot}$, as the mass function, and their semi-major axis of the system, of $3.7 \mathrm{AU}$, as the semi-major axis of the orbit of the Mira component. We also note that not all points listed in their Table 1 were plotted on their figures showing VC versus orbital phase, namely, they did not measurement from Alcolea et al. (1999). In the text, the authors wrote that points from Hollis et al. (2000) are listed in their Table 1, although they were not displayed there. These data points were plotted on the figures showing VC versus Julian days, but they were missing on the figure of $\mathrm{VC}$ versus orbital phase. The authors also did not compare the orbit solution with the resolved image of the binary (Hollis et al. 1997), nor include radial velocity measurements before 1946 (Merrill 1935, 1950). These points were also ignored in all previous papers about the orbit estimation 
Table 1. Radial velocities of R Aqr used for orbital elements determination.

\begin{tabular}{|c|c|c|c|c|}
\hline JD & $\mathrm{RV}\left[\mathrm{km} \mathrm{s}^{-1}\right]$ & Spectral range & Symbol & References \\
\hline 2422255 & -21.6 & Visual & D & Merrill (1935) \\
\hline 2423039 & -22.0 & Visual & D & Merrill (1935) \\
\hline 2429581 & -30.7 & Visual & () & Merrill (1950) \\
\hline 2430336 & -30.2 & Visual & () & Merrill (1950) \\
\hline 2432235.5 & -28.5 & Visual & () & Merrill (1950) \\
\hline 2433019.3 & -29.0 & Visual & () & Merrill (1950) \\
\hline 2441237 & -21.7 & Visual & - & Jacobsen \& Wallerstein (1975) \\
\hline 2442939 & -22.0 & Near-IR & * & Hinkle et al. (1989) \\
\hline 2443527.5 & -26.6 & $v=1, J=1-0$ & + & Lepine et al. (1978), Zuckerman (1979) \\
\hline 2444078 & -25.9 & $v=1, J=1-0$ & - & Cohen \& Ghigo (1980), Spencer et al. (1981), Lane (1982) \\
\hline 2444356 & -27.0 & $v=1, J=1-0$ & $\times$ & Lane (1982) \\
\hline 2444509 & -25.6 & Visual & 0 & Wallerstein (1986) \\
\hline 2444748 & -30.1 & Visual & 0 & Wallerstein (1986) \\
\hline 2445535 & -27.7 & Near-IR & * & Hinkle et al. (1989) \\
\hline 2445574.5 & -27.6 & $v=1, J=1-0$ & $\Delta$ & Cho et al. (1996), Jewell et al. (1991) \\
\hline 2445862 & -30.1 & Visual & 0 & Wallerstein (1986) \\
\hline 2445890.6 & -28.0 & Near-IR & * & Hinkle et al. (1989) \\
\hline 2446378.5 & -27.8 & Near-IR & * & Hinkle et al. (1989) \\
\hline 2447247 & -28.5 & $v=1, J=1-0$ & $\Delta$ & Martinez et al. (1988) \\
\hline 2447338 & -28.9 & Near-IR & * & Hinkle et al. (1989) \\
\hline 2447634 & -28.5 & $v=1, J=1-0$ & $\Delta$ & Martinez et al. (1988) \\
\hline 2447870 & -27.1 & $v=1, J=1-0$ & $\boldsymbol{\nabla}$ & Pardo et al. (2004) \\
\hline 2448240 & -27.0 & $v=1, J=2-1$ & $\bullet$ & Schwarz et al. (1995) \\
\hline 2448283 & -28.3 & $v=1, J=1-0$ & $\boldsymbol{\nabla}$ & Pardo et al. (2004) \\
\hline 2448696 & -27.4 & $v=1, J=1-0$ & $\boldsymbol{\nabla}$ & Pardo et al. (2004) \\
\hline 2448998.4 & -26.4 & $v=1, J=2-1$ & $\bullet$ & Schwarz et al. (1995) \\
\hline 2449435 & -27.0 & $v=1, J=1-0$ & $\boldsymbol{\nabla}$ & Pardo et al. (2004) \\
\hline 2449439 & -26.3 & $v=1, J=2-1$ & $\bullet$ & Schwarz et al. (1995) \\
\hline 2449804 & -27.0 & $v=1, J=1-0$ & $\boldsymbol{\nabla}$ & Pardo et al. (2004) \\
\hline 2450068.8 & -26.8 & $v=1, J=1-0$ & $\diamond$ & Boboltz et al. (1997) \\
\hline 2450407 & -24.0 & $v=1, J=1-0$ & $\square$ & Hollis et al. (2000) \\
\hline 2450948 & -24.0 & $v=1, J=1-0$ & 口 & Hollis et al. (2000) \\
\hline 2451390.4 & -25.6 & $v=1, J=2-1$ & $\Delta$ & Kang et al. (2006), Hollis et al. (2000) \\
\hline 2451785.8 & -23.2 & $v=1, J=2-1$ & $\star$ & Kang et al. (2006) \\
\hline 2451892 & -24.3 & $v=1, J=1-0$ & $\oplus$ & Hollis et al. (2001), Cotton et al. (2004), McIntosh \& Rustan (2007) \\
\hline 2453253 & -23.0 & $v=1, J=1-0$ & $\otimes$ & Cotton et al. (2006) \\
\hline 2453784.3 & -22.7 & $v=1, J=1-0$ & $\nabla$ & McIntosh \& Rustan (2007) \\
\hline 2454112 & -22.8 & $v=1, J=1-0$ & $\nabla$ & McIntosh \& Rustan (2007) \\
\hline
\end{tabular}

from radial velocities (Wallerstein 1986; Hinkle et al. 1989). The authors also neglected radial velocity measurements of the $\mathrm{SiO}$ maser by Zuckerman et al. (1979), Hollis et al. (1986), Hall et al. (1987), Allen et al. (1989), Heske (1989), Jewell et al. (1991), Patel et al. (1992), Gray et al. (1995), Schwarz et al. (1995), Hollis et al. (2000, 2001), Imai et al. (2001), Cotton et al. (2004, 2006), and Kang et al. (2006). We also note that the observations studied by Pardo et al. (2004) included the observations analyzed by Alcolea et al. (1999), which included the data used by Martinez et al. (1988). These three data sets are thus not entirely different, and the later studies were based simply on data collected over longer time.

This paper contains an analysis of the revised radial velocity data listed in Table 1 of McIntosh \& Rustan (2007) and additional velocity data (listed here in Table 1), as well as discussion of the connections between the orbital motion and other phenomena shown by R Aqr.

\section{Orbit determination}

The data from Table 1 of McIntosh \& Rustan (2007) were revised and supplemented by additional radial velocity measurements from Merrill (1935, 1950), Zuckerman (1979), Martinez et al. (1988), Jewell et al. (1991), Schwarz et al. (1995), Hollis et al. (2000, 2001), Cotton et al. (2004, 2006), and Kang et al. (2006).
The blue-violet absorption lines in Mira variables show constant shift of between $-5 \mathrm{~km} \mathrm{~s}^{-1}$ and $-10 \mathrm{~km} \mathrm{~s}^{-1}$ with respect to near-IR velocities (Hinkle et al. 1984; and references therein). In our study, a shift equal to $-6 \mathrm{~km} \mathrm{~s}^{-1}$ was adopted. The blueviolet absorption lines do not show any variation with pulsation period and do not need any additional correction. Absorption lines from the red part of the optical spectrum exhibit significant variation but there is no way of correcting for this effect in a single measurement. For this reason, we did not use three data points from Jacobsen \& Wallerstein (1975), which had been previously used for the spectroscopic orbit determinations by Wallerstein (1986), Hinkle et al. (1989), and McIntosh \& Rustan (2007).

In the near-IR, the radial velocity (RV) variations of $\mathrm{R}$ Aqr were dominated by stellar pulsations $\left(\Delta V \sim 40 \mathrm{~km} \mathrm{~s}^{-1}\right)$. To remove changes due to the pulsations, Hinkle et al. (1989) complete a linear fit to the infrared velocity curve between phase 0.1 and 0.8 , and then adopted as the stellar velocities the residuals remaining after subtracting the velocity-curve fit from each measurement plus the center-of-mass velocity of the Mira of $-29.1 \mathrm{~km} \mathrm{~s}^{-1}$. The value of the center-of-mass velocity was estimated from the CO $\Delta v=3$ velocity at phase 0.36 , following the results of Hinkle et al. (1984). Unfortunately, Hinkle et al. (1989) used an old ephemeris of Campbell (1955). Since R Aqr displays significant variation in its pulsation period (see Fig. 1), 


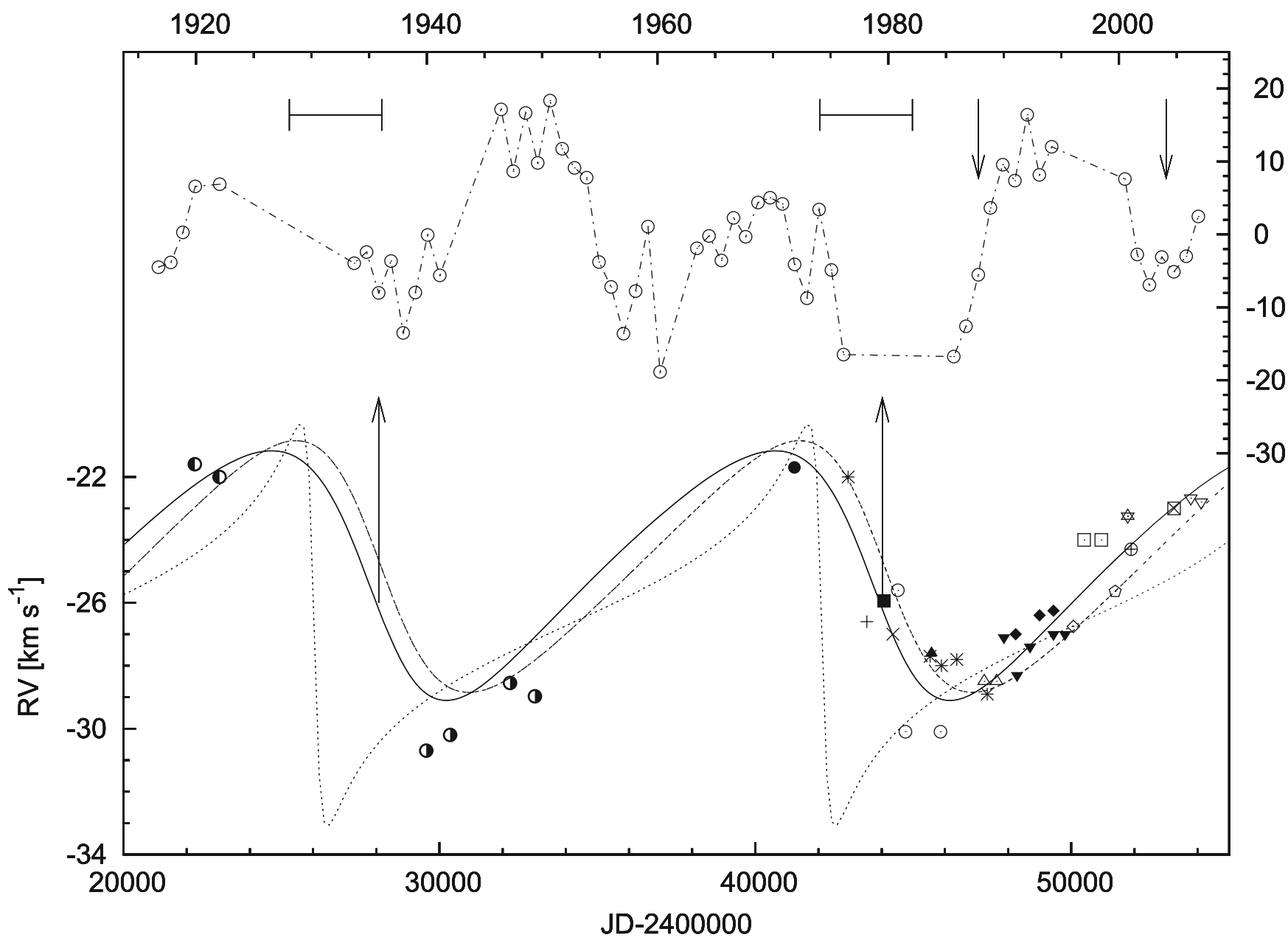

Fig. 1. The O-C diagram (top) and RV variations (bottom) for R Aqr. Observations from different sources are marked like in Table 1. The solid curve represents the fitted orbital elements (Table 2). The dotted curve represents the orbital model proposed by Hollis et al. (1997). The dashed curve represents solution, with modified $\omega=87$ which better fits the VLA observations. Bars show times of hypothetic eclipse, short arrows represent moments of jet ejection (Nichols et al. 2007), whilst long arrows show periastron passage.

we repeated these corrections with the ephemeris $\mathrm{JD}(\max )=$ $2442404.2( \pm 0.4)+388.1( \pm 0.1) \times E$, derived for the $1975-1989$ period covering Hinkle et al. (1989) observations.

To calculate the orbital solution all data points were averaged in 387-day bins corresponding to the pulsation period of the Mira component. The velocity changes due to the orbital motion are negligible during such an interval. The data obtained from different methods were binned separately. Since different maser transitions are formed at different distances from the stellar surface, they were also binned separately. We excluded single measurements for various maser transitions (Allen et al. 1989; Patel et al. 1992; Gray et al. 1995; Imai et al. 2001) obtained for epochs covered by more extensive data sets. In Table 1, we list the radial velocity data used to derive the orbital elements of R Aqr and then converted to local standard of rest $(L S R)$ velocities, which are also plotted in Fig. 1.

We also decided to exclude from our analysis the $\mathrm{SiO}$ maser data obtained during the period JD $2446000-47500$ (data from Hollis et al. 1986; Heske 1989; Alcolea et al. 1999; and Hall et al. 1990) because the maser emission showed at that time significantly different behaviour e.g. a few fainter emission, irregular contours than in later epochs, as well as the masers in isolated Miras (see Pardo et al. 2004). The $\mathrm{SiO}$ masers in R Aqr display a ring-like morphology $\sim 31$ mas $(\sim 6.2[d / 200$ pc] AU) in diameter (Boboltz et al. 1998). So, these masers lie very close to the stellar surface, namely at a distance of only $\sim 1.5$ radii from the Mira (see Sect. 3) in a region dominated by stellar pulsations and permeated by circumstellar shocks. In addition, the $\mathrm{SiO}$ masers can be seriously affected by tidal interactions during the periastron passage when the size of the $\mathrm{SiO}$ maser region became comparable to the Roche lobe radius of the Mira. In fact, according to our orbital solution (see below) the Alcolea et al. (1999) observations were obtained around periastron. The deep minimum in both VCs and flux of maser emission that appeared around JD $2446700-47300$, coincided with the jet formation (Nichols et al. 2007) and a rise in the UV continuum (Meier \& Kafatos 1995). We suspect that the $\mathrm{SiO}$ masers may have been partly disrupted by rising UV radiation during the jet formation, and tidal interaction during the periastron passage and then gradually rebuild.

To identify the spectroscopic orbit, we first estimated a period of RV variations of 43.6 yr using Ortfit (based on the method of Schwarzenberg-Czerny 1996), and then calculated the orbital parameters: $T_{0}$, the time of periastron passage, $\gamma$, the baricentral velocity, $K$, the semi-amplitude, $\omega$, the longitude of periastron, and $e$, the eccentricity with fixed period. We applied Bertiau's program (1967) based on the Lehmenn-Filhés method. As a result, we obtained an eccentric, $e=0.25 \pm 0.07$, orbit with reasonable errors (Table 2). Figure 2 displays the data and the best-fit velocity curve versus orbital phase. 


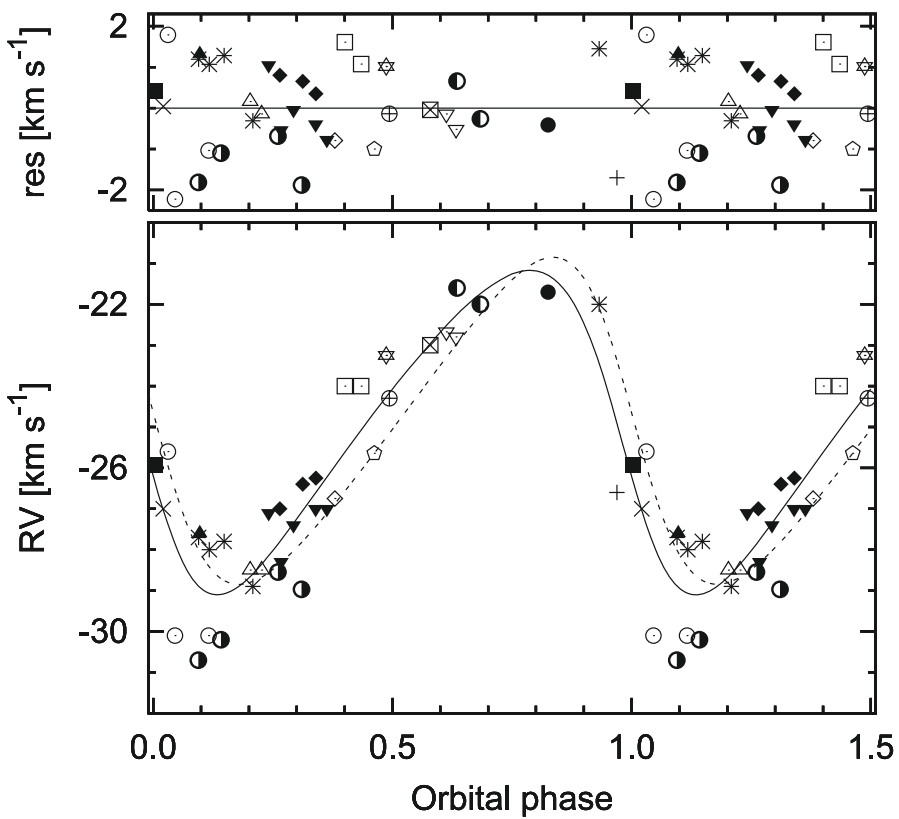

Fig. 2. Bottom: the RV data and fitted orbital curve (Table 2) against orbital phase. Symbols are the same as in Fig. 1. The solid curve represent our orbital solution. The dashed curve represents solution, with modified $\omega=87$ which better fits the VLA observations. Top: difference between the RV data and our orbital fit (solid line in the lower pannel).

Table 2. Orbital elements for R Aqr.

\begin{tabular}{lc}
\hline \hline Element & Value \\
\hline$P_{\text {orb }}($ day $)$ & $15943 \pm 471$ \\
$\gamma\left(\mathrm{km} \mathrm{s}^{-1}\right)$ & $-24.9 \pm 0.2$ \\
$K\left(\mathrm{~km} \mathrm{~s}^{-1}\right)$ & $4.0 \pm 0.4$ \\
$e$ & $0.25 \pm 0.07$ \\
$\omega(\mathrm{deg})$ & $106 \pm 19$ \\
$T_{0}$ & $2444019 \pm 728$ \\
$\Sigma(\mathrm{O}-\mathrm{C})^{2}$ & 40 \\
$\sigma\left(\mathrm{km} \mathrm{s}^{-1}\right)$ & 1.11 \\
\hline
\end{tabular}

Figure 1 also compares the RV variations with the $\mathrm{O}-\mathrm{C}$ data for the Mira pulsations based visual data collected by amateur observers from AAVSO. The $\mathrm{O}-\mathrm{C}$ is a plot showing the difference between the observed $(\mathrm{O})$ times of maximum light and the values calculated according to an adopted ephemeris $(\mathrm{C})$ as a function of time. A description of the $\mathrm{O}-\mathrm{C}$ method can be found in a review by Zhou (2003). To derive a new pulsation ephemeris, we first estimated the time of each maximum in the light curve by fitting a third-order Fourier polynomial. Then a linear ephemeris:

$$
\mathrm{JD}(\max )=2416070 \pm 4+387.30 \pm 0.07 \times E
$$

was fitted to the obtained times of maxima. The resulting $\mathrm{O}-\mathrm{C}$ diagram shows a parabolic shape that corresponds to an increase in the period rate of $0.013 \pm 0.002$ day per cycle. After subtracting this parabolic trend, a sinusoidal variation with a period of 22.5 years and amplitude of about 8 days (Fig. 1) is visible.

\section{Discussion}

Our orbital solution yields the semi-major axis of the Mira of

$$
a_{\mathrm{g}} \sin i=\frac{K P}{2 \pi} \sqrt{1-e^{2}}=5.68_{-0.83}^{+0.85} \mathrm{AU}
$$

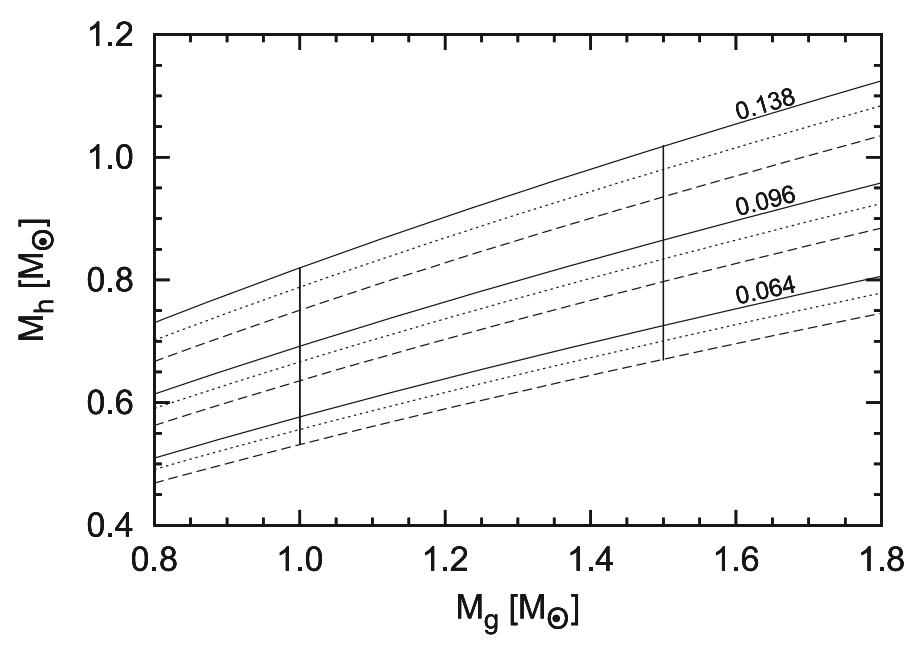

Fig. 3. The permitted component masses constrained by the observed mass function as well as its maximum and minimum values (solid lines labeled with the values of $f(M)$ ), and assuming $i=70^{\circ}$. Dotted and dashed lines show the solution for $i=75^{\circ}$, and $i=90^{\circ}$, respectively.

and the mass function of

$f(\mathrm{M})=\frac{1}{2 \pi \mathrm{G}} \cdot \mathrm{PK}^{3}\left(1-\mathrm{e}^{2}\right)^{3 / 2}=\frac{\left(M_{\mathrm{h}} \sin \mathrm{i}\right)^{3}}{\left(M_{\mathrm{h}}+M_{\mathrm{g}}\right)^{2}}=0.096_{-0.032}^{+0.042} M_{\odot}$,

where $M_{\mathrm{g}}$, and $M_{\mathrm{h}}$ represent masses of the giant and the hot component, respectively, and the errors are set by the uncertainties in the orbital parameters (see Table 2). The mass function sets a lower limit to the hot companion mass.

For further discussion, we assume that the symbiotic binary R Aqr consist a Mira variable and a white dwarf (WD) companion with an orbit inclined at $i=70^{\circ}\left(\sim 72^{\circ}\right.$ according to Solf \& Urlich 1985). Assuming plausible components masses, $M_{\mathrm{g}}=1-$ $1.5 M_{\odot}$, and $M_{\mathrm{h}}=0.5-1.4 M_{\odot}$, for the Mira and the WD, respectively, the total mass of system $M_{\text {tot }}=M_{\mathrm{h}}+M_{\mathrm{g}}$ should be within the range $1.5-3 M_{\odot}$. The component masses are also limited by the orbital solution. Therefore, if we consider $f(M)=0.064-$ $0.138 M_{\odot}$ and $M_{\mathrm{g}}=1-1.5 M_{\odot}$, we derive $M_{\mathrm{tot}}=1.6-2.5 M_{\odot}$, $M_{\mathrm{h}}=0.57-1.02 M_{\odot}$, and the mass ratio $q=M_{\mathrm{g}} / M_{\mathrm{h}}=1.2-2.1$ (Fig. 3). These values are in good agreement with the commonly adopted model of symbiotic binaries. The semi-major axis of the system, $a$, is $14.2-16.8 \mathrm{AU}$, which corresponds to 71-84 mas on the sky for a distance of $d=200 \mathrm{pc}$ (see below).

Our orbital solution can be tested and refined by using resolved observations of R Aqr (Hege et al. 1991; Hollis et al. 1997). In particular, the binary components of R Aqr were resolved by VLA observations (Hollis et al. 1997) obtained on 20 Nov. 1996 (JD = 2450407 ). The measured separation between the VLA image in $\mathrm{SiO}$ maser $v=1, J=1-0$ transition and the continuum emission in a $50 \mathrm{MHz}$ bandwidth at $43.165 \mathrm{GHz}$ associated with an HII region surrounding the hot companion was $\rho=55 \pm 2$ mas $(11[d / 200 \mathrm{pc}] \mathrm{AU})$, and the position angle was $\theta=18^{\circ} \pm 2^{\circ}$. This can be compared with the projected component separation on the sky plane calculated from our orbital elements. The orbital elements (Table 2) combined with the orbital inclination of $i=70^{\circ}$ (Solf \& Urlich 1985) inferred the projected separation of $\rho=0.44 a \sim 31-37[d / 200 \mathrm{pc}]^{-1}$ mas, and the difference between the apparent binary position angle and the position angle of the line of nodes (the binary orientation on sky) of $\theta-\Omega=-109^{\circ}$.

Our predicted component separation on the sky is consistent with the VLA observations only if the distance is somewhat 


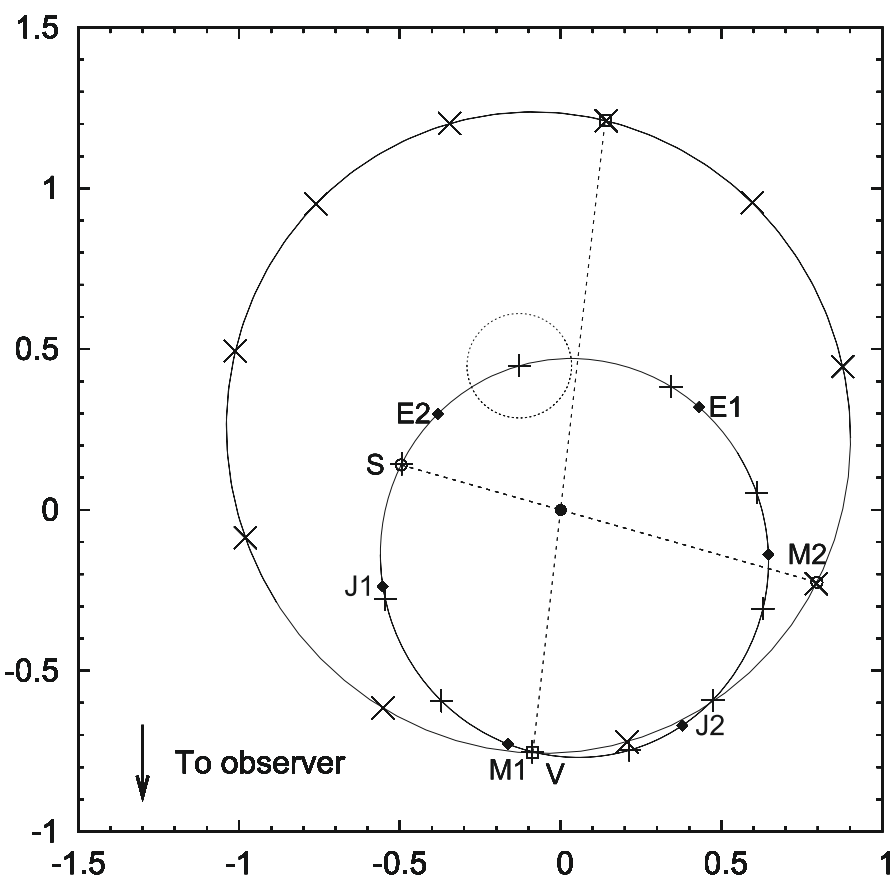

Fig. 4. The orbit of the Mira (+) and hot $(\times)$ component in the R Aqr binary system in steps of $\Delta \phi=0.1$. In this representation, the stars move anti-clockwise. The dotted circle represents the Mira boundary at $\phi=0$ (periastron passage). The solid dot marks the mass center. Axes are in units of the semi-major axis $a$. The positions of Mira at the beginning and end of eclipse is marked by E1, and E2, respectively whereas the main and secondary maxima in $\mathrm{O}-\mathrm{C}$ diagram are denoted by $\mathrm{M} 1$ and M2, respectively. J1 and J2 mark the two jet ejection episodes. Open squares connected with dotted line and marked by $\mathrm{V}$ show the component position during the VLA observation (Hollis et al. 1997). Open dots connected with dotted line and marked by S show the component position during the speckle observation (Hege et al. 1991).

lower than $200 \mathrm{pc}$ or we underestimate the $\rho$ value due to uncertainties in our orbital elements.

The predicted value of $\rho$ should in fact increase, changing $\omega$ (see also Fig. 4), and/or increasing $e$, and/or decreasing $T_{0}$. For example, $\rho$ should increase to $0.65 a\left(46-55\left[d / 200 \mathrm{pc}^{-1}\right.\right.$ mas) just by adopting a lower value of $\omega=87$, i.e. lower only by an amount approximately equal to the standard-deviation uncertainty of $\omega$.

The distance to R Aqr is also relatively uncertain, and published estimates based on various methods are in the range 180260 pc (e.g. 180 pc by Solf \& Ulrich $1985 ; 181$ pc by Lepine et al. $1978 ; 240$ pc using the most recent period-luminosity relation from Whitelock et al. 2008; and 260 pc by Baade 1943, 1944). The Hipparcos parallax of R Aqr is $5.07 \pm 3.15$ mas (Perryman et al. 1993), which corresponds to a distance of $197_{-75}^{+323} \mathrm{pc}$. Our determination of the parameters of the spectroscopic orbit is thus in a good agreement (within $\leq 1-\sigma$ errors in the orbital parameters) with those inferred from the VLA resolved observations. The value of $\theta=18^{\circ}$, measured with the VLA, implies that $\Omega=127^{\circ}$. Thus, the position of the binary orbit on the sky reproduces well the general picture of the nebula around R Aqr, i.e. the orbital plane is perpendicular to the jets (see Fig. 7 in Solf \& Urlich 1985).

The orbital parameters derived for the speckle observations (Hege et al. 1991) completed on 16 Oct. 1983 are inconsistent with our orbit in any case, because the measured separation, $\rho=$ $124 \pm 2$ mas which corresponding to $25[d / 200 \mathrm{pc}] \mathrm{AU}$, is too large for any orbital phase. Hege et al. (1991) probably did not detect the Mira companion but rather an $\mathrm{H} \alpha$ emission region in the SW counterjet.

Our orbital solution predicts the times of spectroscopic conjunctions at $T_{\text {conj I }}=2450859$ (Feb. 1998), $T_{\text {conj II }}=2443658$ (May 1978) for the inferior (the Mira is in front of the hot component), and the superior (the Mira behind) conjunction, respectively. The superior conjuction coincides with the last dust obscuration/eclipse (see also Fig. 4). Although the conjunction times could differ by up to 1-2 years due to uncertainties in the orbital parameters, especially $T_{0}, e$, and $\omega$, the obscuration lasted about 6 years, and the coincidence between these two events is obvious.

Adopting plausible binary parameters, i.e. the mass ratio $q \sim 1.6, M_{\mathrm{tot}}=2 M_{\odot}$, and $a=15 \mathrm{AU}$ (see above), we estimate the minimum component separation to be $a(1-e) \sim 11 \mathrm{AU}$, and the Roche lobe radius for the Mira, to be $R_{\mathrm{L}} \sim 4 \mathrm{AU}$. The near-IR interferometry of R Aqr gave the diameter of the Mira expressed as a diameter of uniform disk, $\Theta_{\mathrm{UD}}=14.06-20.8 \mathrm{mas}$ in $K, 17.7$ mas in $J$, and 17.7-19.07 mas in $H$, in intermediate and minimum pulsation phase, respectively (van Belle et al. 1996; Millan-Gabet et al. 2005; Ragland et al. 2006). These values correspond to the average radius of the Mira component, $R_{\mathrm{g}} \sim 2$ AU. Even during periastron passage, the Mira variable therefore remains relatively far from filling the Roche lobe.

Many detached interacting binaries, including the symbiotic binaries as well as many X-ray binaries show evidence for much higher mass transfer rate than predicted by models of spherically symmetric winds. This phenomenon can be accounted for by wind focused towards the compact component. Podsiadlowski $\&$ Mohamed (2007) proposed a model for $o$ Cet binary system that can explain this focusing. In their model, a slow wind from Mira fills its Roche lobe and then the matter falls - via the $L_{1}$ Lagrangian point - into an accreting stream onto an accretion disk around the companion. This model is even more relevant to R Aqr where the component separation is significantly smaller than that in $o$ Cet. In particular, it could explain the increase in extinction towards the Mira during the last "eclipse" in 1974-1981 caused by a neutral material in the accreting stream. According to our orbital solution, the streaming material can obscure the Mira at that time, and in addition, the proximity of periastron (see Fig. 4) could give rise to enhanced mass transfer. A similar enhanced, wind-obscuration scenario for R Aqr was discussed by Mikołajewska \& Kenyon (1992), although in that case the source of the increased mass loss was proposed to be a helium flash above a massive core $\left(M_{\text {core }} \sim 1.36 M_{\odot}\right)$ of the Mira.

The orbital configuration of R Aqr could also explain the observations of the $\mathrm{OH}$ and $\mathrm{H}_{2} \mathrm{O}$ masers that originate in circumstellar regions more distant from the Mira variable (e.g. $\mathrm{H}_{2} \mathrm{O}$ masers zone is 10-20 AU) than the $\mathrm{SiO}$ maser emission region. The $\mathrm{OH}$ and $\mathrm{H}_{2} \mathrm{O}$ maser lines were not detected in 1979 and 1984 (Cohen \& Ghigo 1980; Norris et al. 1984) when the hot companion was in front of Mira. Ivison et al. (1994) reported detection of weak $\mathrm{OH}$ and $\mathrm{H}_{2} \mathrm{O}$ maser emission in 1993, when the Mira was in front of the hot companion and the masers were shielded from its radiation. They claimed, however, that the $\mathrm{OH}$ maser detection was only tentative, and that the velocity measurement was very unusual (an expansion velocity of $10 \mathrm{~km} \mathrm{~s}^{-1}$ is rather large for this system), and only the red peak should have been detected, and additional spectra obtained using the VLA during 1994 June did not show the $\mathrm{OH}$ maser line (Ivison et al. 1998). The $\mathrm{H}_{2} \mathrm{O}$ line appeared more promising, although weak for a Mira variable with relatively strong $\mathrm{SiO}$ masers. It was also clearly detected during 3 epochs in 1993-1995 
(Ivison et al. 1998). Ivison et al. (1998) proposed three possible mechanisms that could inhibit the $\mathrm{OH}$ and $\mathrm{H}_{2} \mathrm{O}$ maser formation in their natural environment, involving either the orbital motion of the companion or its UV radiation and fast wind, or line obscuration at low frequencies by optically thick, ionized gas.

The apparent correlation between the orbital position and the $\mathrm{O}-\mathrm{C}$ for the Mira pulsations is extremely interesting. In particular, minima in the $\mathrm{O}-\mathrm{C}$ diagram occur around the periastron (see Fig. 1). This may be due to some distortion of the Mira caused by tidal interaction during the periastron passage, for example if the Mira became elongated towards the hot component, the pulsation may need more time for propagation in this direction. According to our orbital model, the main maximum of $\mathrm{O}-\mathrm{C}$ appears close to the inferior conjunction, when the Mira eclipses the hot companion, whereas the secondary, lower maximum appears in the middle of the two conjunctions, when both components are well separated and we can see the elongated side.

Finally, the orbital solution proposed is inconsistent with the $17 \mathrm{yr}$ orbital period for R Aqr proposed by Nichols et al. (2007). They noticed that radio observations obtained in 1987 and 2004 exhibited non-thermal spectra, which are usually interpreted to be a signature of a newly emitted jet (in stellar sources older jets are generally thermal), suggested that the jet formation is coupled with periastron. In our opinion, such a short period appears unrealistic not only because it is not reflected by the RV variation but also because in that case there would not be sufficient room for a Mira with a dust envelope. The 17-yr period implies a semimajor axis of $\sim 8 \mathrm{AU}$, whereas the minimum component separation for D-type symbiotic binaries is about 10-15 AU, which for typical masses (see above) indicates orbital periods longer than 20 yrs (Mikołajewska 1999). It appears that the jet ejection in R Aqr connot be connected with the orbital motion.

\section{Conclusions}

Based on published radial velocities of its Mira component, we have derived new orbital parameters for the symbiotic binary R Aqr. We have found in particular, the orbital period of $43.6 \mathrm{yr}$, and showed that the mass function is consistent with the presence of a typical, $1-1.5 M_{\odot}$, Mira variable accompanied by a 0.6-1 $M_{\odot}$ white dwarf. We also showed that our spectroscopic orbit is consistent with the VLA astrometry (Hollis et al. 1997) and inconsistent with the speckle interferometery (Hege et al. 1991). Our orbital model allow us to interpret the "eclipses" of Mira as obscuration by the accretion stream. We also showed that the jet ejection (Nichols et al. 2007) is not connected with the orbital position. Finally, we note that September 2012 should be a perfect time to complete resolved imaging of $\mathrm{R}$ Aqr, since the Mira component will then be at minimum brightness and the separation between the components on sky will be close to its maximum possible value.

Acknowledgements. This study made use of the American Association of Variable Star Observers (AAVSO) International Database contributed by observers worldwide. We are grateful to the anonymous referee for valuable comments. We also thank Radek Smolec for comments to the first version of this paper and Wojtek Pych for providing the Ortfit software. This work was partly supported by the Polish Research grant N203 395534.

\section{References}

Alcolea, J., Pardo, J. R., Bujarrabal, V., et al. 1999, A\&AS, 139, 461 Allen, D. A., Hall, P. J., Norris, R. P., et al. 1989, MNRAS, 236, 363
Baade, W. 1943, Carnegie Inst. Washington Yearb., 42, 17 Baade, W. 1944, Carnegie Inst. Washington Yearb., 43, 12 Bertiau, F. C. 1967, IAUS, 30, 227

Boboltz, D. 1997, Ph.D. Thesis, Virginia Polytechnic Univ. Boboltz, D., Diamond, P. J., \& Kemball, A. J. 1998, ASPC, 144, 2438 Campbell L. 1955, Studies of Long Period Variables, AAVSO Pub.

Cho, S.-H., Kaifu, N., \& Ukita, N. 1996, A\&AS, 115, 117 Cohen, N. L., \& Ghigo, F. D. 1980, AJ, 85, 451

Cotton, W. D., Mennesson, B., Diamond, P. J., et al. 2004, A\&A, 414, 275

Cotton, W. D., Vlemmings, W., Mennesson, B., et al. 2006, A\&A, 456, 339

Gray, M. D., Ivison, R. J., Yates, J. A., et al. 1995, MNRAS, 277, 67

Gudehus, D. 2001, BAAS, 33, 850

Hall, P. J., Robin, M., Wark, R. M., \& Wright, A. E.1987, Proc. Astron. Soc. Aus., 7, 50

Hall, P. J., Allen, D. A., Troup, E. R., Wark, R. M., \& Wright, A. E. 1990, MNRAS, 243, 480

Hege, E. K., Allen, C. K., \& Cocke, W. J. 1991, ApJ, 381, 543

Heske, A. 1989, A\&A, 208, 77

Hinkle, K., Scharlach, W., \& Hall, D. N. 1984, ApJS, 56, 1

Hinkle, K., Wilson, T., Scharlach, W., \& Fekel, F. 1989, AJ, 98, 1820

Hollis, J. M., Michalitsianos, A. G., Kafatos, M., Wright, M. C. H., \& Welch, W. J. 1986, ApJ, 309, L53

Hollis J. M., Pedelty, J. A., \& Lyon, R. G. 1997, ApJ, 482, L85

Hollis, J. M., Pedelty, J. A., Forster, J., et al. 2000, ApJ, 543, L81

Hollis, J., Boboltz, D., Pedelty, J., White, S., \& Forster, J. 2001, ApJ, 559, L37

Ivison, R. J., Seaquist, E. R., \& Hall, P. J. 1994, MNRAS, 269, 218

Imai, H., Miyoshi, M., Ukita, N., et al. 2001, PASJ, 53, 259

Ivison, R. J., Yates, J. A., \& Hall, P. J.1998, MNRAS, 295, 813

Jacobsen, T. S., \& Wallerstein, G. 1975, PASP, 87, 269

Jewell, P. R., Snyder, L. E., Walmsley, C. M., Wilson, T. L., \& Gensheimer, P. D. 1991, A\&A, 242, 211

Kang, J., Cho, S.-H., Kim, H.-G., et al. 2006, ApJS, 165, 360

Kellogg, E., Anderson, C., Korreck, K., et al. 2007, ApJ, 664, 1079

Lane, A. 1982, Ph.D. Thesis, Univ. Massachusetts

Lepine, J., LeSqueren, A., \& Scalise, E. 1978, ApJ, 225, 869

Martinez, A., Bujarrabal, V., \& Alcolea, J. 1988, A\&AS, 74, 273

McIntosh, G. C. 2006, AJ, 132, 1046

McIntosh, G. C., \& Rustan, G. 2007, AJ, 134, 55

Meier, S. R., \& Kafatos, M. 1995, ApJ, 451, 359

Merrill, P. W. 1935, ApJ, 81, 312

Merrill, P. W. 1950, ApJ, 112, 514

Mikołajewska, J. 1999, in Optical and Infrared Spectroscopy of Circumstellar

Matter, ed. E. Guenther, \& B. Stecklum, ASP Conf. Ser., 188, 291

Mikołajewska, J., \& Kenyon, S. J. 1992, MNRAS, 256, 177

Millan-Gabet, R., Pedretti, E., Monnier, J. D., et al. 2005, ApJ, 620, 961

Nichols, J. S., DePasquale, J., Kellogg, E., et al. 2007, ApJ, 660, 651

Norris, R. P., Haynes, R. F., Wright, A. E., \& Allen, D. A. 1984, Proc. Astron.

Soc. Aust., 5, 562

Pardo, J. R., Alcolea, J., Bujarrabal, V., et al. 2004, A\&A, 424, 145

Patel, N. A., Joseph, A., \& Ganesan, R. 1992, J. Astrophys. Astron., 13, 241

Perryman, M. A. C., Lindegren, L., Kovalevsky, J., et al. 1997, A\&A, 323, 49

Posiadlowski, Ph., \& Mohamed, S. 2007, in Evolution and chemistry of

symbiotic star, binary post-AGB and related objects, ed. J Mikołajewska, \&

R. Szczerba, Baltic Astronomy, 16, 26

Ragland, S., Traub, W. A., Berger, J.-P., et al. 2006, ApJ, 652, 650

Schwarz, H. E., Nyman, L.-A., Seaquist, E. R., \& Ivison, R. J.1995, A\&A, 303, 833

Schwarzenberg-Czerny, A. 1996, ApJ, 460, L107

Solf, J., \& Ulrich, H. 1985, A\&A, 148, 274

Spencer, J., Winnberg, A., Olnon, F., et al. 1981, AJ, 86, 392

Townley, S. D., Cannon, A. J., \& Campbell, L. 1928, Ann. Harv. Coll. Obs., 79, 161

van Belle, G. T., Dyck, H. M., Benson, J. A., \& Lacasse, M. G. 1996, AJ, 112, 2147

Wallerstein, G. 1986, PASP, 98, 118

Whitelock, P. A. 2003, in Symbiotic Stars Probing Stellar Evolution, ed. R. L. M.

Corradi, J. Mikołajewska, \& T. J.Mahoney, ASP Conf. Ser., 303, 41

Whitelock, P. A., Feast, M. W., Catchpole, R. M., Carter, B. S., \& Roberts, G. 1983, MNRAS, 203, 351

Whitelock, P. A., Feast, M. W., \& van Leeuwen, F. 2008, MNRAS, 386, 313

Willson, L. A., Garnavich, P., \& Mattei, J. A. 1981, Inf. Bull. Variable Stars, 1961, 1

Zhou, A. 1999, Publ. Beijing Astron. Obs., 33, 17

Zuckerman, B. 1979, ApJ, 230, 442 